\title{
Germanica
}

\section{L'exotisme fantastique de Hanns Heinz Ewers}

Exatik und Phantastik bei H.H. Ewers

Jean-Jacques Pollet

\section{OpenEdition}

Journals

Édition électronique

URL : http://journals.openedition.org/germanica/2805

DOI : 10.4000/germanica.2805

ISSN : 2107-0784

\section{Éditeur}

Université de Lille

\section{Édition imprimée}

Date de publication : 30 juin 1987

Pagination : 49-65

ISSN : 0984-2632

\section{Référence électronique}

Jean-Jacques Pollet, « L'exotisme fantastique de Hanns Heinz Ewers », Germanica [En ligne], 1 | 1987 mis en ligne le 20 octobre 2015, consulté le 06 octobre 2020. URL : http://journals.openedition.org/ germanica/2805; DOI : https://doi.org/10.4000/germanica.2805

Ce document a été généré automatiquement le 6 octobre 2020.

(C) Tous droits réservés 


\section{L'exotisme fantastique de Hanns Heinz Ewers}

Exatik und Phantastik bei H.H. Ewers

Jean-Jacques Pollet

\section{NOTE DE L'AUTEUR}

N.B. : L'étude à ce jour la plus complète, sur H.H. Ewers. est le livre de Michael Sennewald, Hanns Heinz Ewers. Phantastik und Jugendstil, Verlag Anton Hain, Meisenheim. 1973.

Taumelnd, wie ein Trunkener, schwankte er dem Hause zu... «Ich will nach Hause»,

flüsterte er. «Die Muntter wartet».

H.H. Ewers, Alraune (derniers mots)

Le héros fantastique, dans les grands classiques du genre du XIXe siècle, est en général le contraire d'un aventurier. Il est confiné dans l'espace clos de son univers propre, auquel il ne peut, ne veut échapper. C'est souvent quelqu'un qui, littéralement, " ne sort pas de chez lui »: le comte d'Athol (Véra, de Villiers de l'Isle-Adam), "après l'affreuse cérémonie du caveau familial », s'enferme, seul, dans "la chambre veuve »; Roderick Usher (The Fall of the House Usher, de B.A. Poe), bien qu'il fût conscient de la fatale influence des lieux sur son esprit, «n'avait jamais osé sortir de ce manoir depuis plusieurs années "; enfin à peine le narrateur du Horta de Maupassant a-t-il fait quelques pas dans la campagne environnante qu'il éprouve « ce besoin douloureux de rentrer qui vous oppresse quand on a laissé au logis un malade aimé et que le pressentiment vous saisit d'une aggravation de son mal... » Pour aucun d'entre eux, il n'y a d'autres cieux. Leur opposera-t-on Melmoth, l'homme errant, de C.R. Maturin, qui, lui, parcourt le monde? Pas vraiment, dans la mesure où la diffusion spatiale, ici, n'est que le faire-valoir de la continuité de l'argument: les déplacements du personnage ne servent qu'à démontrer que, où qu'il aille, il ne pourra échapper à la fatalité qui pèse 
sur lui, qui est irrémédiablement attachée à ses pas ; ses pérégrinations ne sont pas des aventures : elles le ramènent toujours au Même. Les changements de décor ne sont là, au fond, que pour illustrer l'impossibilité de changer de lieu. En littérature fantastique, il n'y aurait ainsi ni partir, ni revenir. C'est le voyage inutile. Le dépaysement interdit.

Généralisation abusive? Nous voulons l'éprouver en regardant délibérément vers des cas singuliers qui dérogent à l'usage, sinon à la règle. Chercher des livres reconnus, admis dans la bibliothèque fantastique et où (pourtant ?) l'on part à la rencontre d'un Ailleurs. Un modèle en la matière est le roman de Poe, The Narrative of Arthur Gordon Pym (1838), récit « d'incroyables aventures et découvertes » à travers « les mers du Sud, les îles et l'océan antarctique ». Au sein de la littérature fantastique allemande du début du XXe siècle, qui nous intéresse plus particulièrement, quelques-uns se situent dans cette lignée ${ }^{1}$. Si les lettres allemandes de l'époque, comme l'a montré Wolfgang Reif, voient la fortune parallèle du genre fantastique et $\mathrm{du}$ roman dit "exotique» ${ }^{2}$ proximité affichée parfois dans la présentation, la distribution des textes à l'intérieur des collections, comme par exemple l'anthologie de nouvelles intitulée seltsame Begebenheiten. Eine Sammlung merkwürdiger Geschichten $(1918)^{3}$ - on peut établir quelques cas de « complicité ». H.H. Ewers en fait partie.

Ce type de fiction invite à analyser comment s'opère le détournement fantastique du thème de l'Ailleurs. Qu'est-ce qui est ici échangé, par rapport à l'image commune de la réalité? Comment cet échange peut-il entrer dans une stratégie narrative de " l'inquiétante étrangeté»? Prélude-t-il enfin à un « retour chez soi » (au propre et au figuré), c'est-à-dire à une réconciliation (à quel prix ?) avec les valeurs familières ?

\section{La scène sauvage}

Maint récit de H.H. Ewers se joue dans un cadre exotique ${ }^{4}$ : les pays méditerranéens, l'Orient, les Îles, les Amériques... Ce ne sont d'ailleurs pas là, pour l'auteur, que voyages en imagination. Dès qu'il eut abandonné la carrière juridique et conquis quelque notoriété en collaborant au «Ueberbrettl» de E. von Wolzogen (1901), H.H. Ewers se mua en écrivain globe-trotter, parcourant le monde (et monnayant, éventuellement, ses «impressions de voyage», comme avec Indien und $I c h^{5}$ ) pour ne revenir qu'épisodiquement à Düsseldorf, chez sa mère, ou passer quelque temps dans son piedà-terre berlinois. Il ne rentra définitivement "chez lui » - nous verrons ce qu'il en coûte - qu'en 1920. Il écrivit ainsi la majorité de ses récits aux quatre coins du monde, ceux-ci portant d'ailleurs pour la plupart, dans l'édition, la signature du lieu où ils furent composés : Capri, Grenade, Rio de Janeiro, Port-au-Prince, etc...

Si l'on peut convenir que toutes les fictions exotiques de H.H. Ewers méritent, globalement et sommairement, d'être qualifiées « d'histoires terrifiantes », il apparaît à l'analyse qu'elles révèlent deux factures différentes, pour ne pas dire contraires, et que la césure se situe avec le conflit mondial et la dernière pièce de la trilogie romanesque du personnage de Frank Braun, Vampir. Ein verwildeter Roman in Fetzen und Farben ${ }^{6}$.

6 Les fictions exotiques qui entrent dans les premiers recueils de nouvelles de H.H. Ewers - Das Grauen (1908), Die Besessenen (1909) - sont construites sur le même modèle, autour 
d'une « scène sauvage » dont l'enjeu, sous la variété des décors, reste identique. Il faut que cette scène se passe devant témoin. Le personnage qui assume cette fonction répond, d'un texte à l'autre, au même portrait. C'est un Européen, prêt à revendiquer éventuellement, avec plus ou moins de véhémence ou d'humour, sa nationalité allemande. Cette appartenance culturelle du témoin sera la mesure du terrifiant: ce qu'il découvre dans son voyage est pour lui (et pour le lecteur censé s'identifier à lui en vertu d'une origine commune) littéralement inouï, inconcevable, par rapport à son image de la réalité, sa propre échelle de valeurs. Mais ce décalage n'est pas pour autant responsable, à lui seul, de la qualité «fantastique » du récit. On pourrait imaginer, en effet, que le spectacle "sauvage», "primitif» soit un simple objet de curiosité ethnographique et le conforte finalement, sous une réaction d'indignation mêlée d'effroi, dans ses propres convictions, le persuade de la supériorité de sa vision du monde d'homme "civilisé ». Or c'est le contraire qui attend le héros ewersien. Il se passe quelque chose entre le témoin et la violence qui s'offre à ses yeux. La découverte de l'Autre, de manière plus ou moins insidieuse, vient le menacer dans son identité, fait vaciller certitudes et croyances héritées. C'est là, précisément, que se joue la conjonction entre exotisme et fantastique. L'enjeu de la "scène sauvage » est bien la contestation de l'image convenue (et convenable) de la réalité véhiculée dans le code de vraisemblance en vigueur pour le lecteur. On peut suivre, d'une histoire à l'autre, quelques ruses de la dramaturgie mise en place à cette fin par H.H. Ewers.

Die Tomatensauce ${ }^{7}$ se passe en Espagne, à Grenade exactement. Le narrateur relate une corrida, puis un combat de coqs auxquels il vient d'assister. Il n'était là, nous dit-il, « au premier rang, que pour prendre des photographies»-indice qui doit signer son détachement, par ailleurs affiché dans la description glacée qu'il nous livre, et qui n'épargne aucun détail de l'horrible, depuis «les chevaux empêtrés dans leurs entrailles sanguinolentes traînant sur le sable de l'arène ", jusqu'aux soubresauts des volatiles qui se tailladent et s'aveuglent à coups de bec et d'ergots. Il n'avoue (ne veut avouer), pour sa part, aucune prédilection particulière pour ce genre de spectacle. C'est un autre étranger, qu'il rencontre par hasard dans le public - et dont il apprend qu'il est le pasteur de la communauté anglaise de la ville - qui lui confesse "la jouissance » qu'il éprouve en la circonstance. Celui-ci invite bientôt le narrateur à l'accompagner à un spectacle plus cruel encore, une "salsa", sorte de duel à mort entre deux adversaires armés de navajas. Et là, pour la première fois apparemment, le narrateur, devant l'insoutenable barbarie du combat, va perdre son impassibilité :

Es war, als ob sich plötzlich ein roter Blutnebel um meine Sinne legte; ich sah, hörte nichts mehr ich versank in ein purpurnes, unergründlich tiefes Meer. Blut drang mir in Ohren und Nase, ieh wollte schreien, aber wie ich den Mund öffnete, füllte er sich mit dickem, warmem Blute ${ }^{8}$.

8 Contrastant avec cette réaction, celle du pasteur, qui va s'agenouiller auprès du corps agonisant de la victime et, plongé dans une sorte d'extase, caresse «de ses mains délicates et fines des mains de femme » les chairs meurtries...

Die Tomatensauce est donc bien l'histoire d'une perversité, et non pas, comme l'a prétendu H. Krüger-Welf, le premier biographe de H.H. Ewers, avant tout soucieux de préserver la réputation de l'écrivain, un document ethnographique sur les mœurs andalouses". La perversité n'est pas du côté des acteurs de la "salsa", ni même du public indigène, mais de celui du spectateur-voyeur étranger. La scène sauvage, en révélant chez ce dernier l'irrésistible fascination du sang, fait craquer le vernis de l'homme cultivé et raffiné qu'il est par ailleurs - fêlure d'autant plus grave, plus 
redoutable qu'il s'agit de quelqu'un qui, par sa fonction ecclésiastique, devrait être l'incorruptible gardien de la morale. Sans doute le narrateur, qui prend soin de marquer sa différence et d'afficher sa nationalité allemande "Je suis, à l'étranger, toujours nationaliste. Je l'ai appris des Anglais depuis longtemps. Right or wrong my contry !») n'est-il pas, apparemment, le plus pervers, face à l'inconduite du pasteur qui rejoint la tradition, dans ce que Mario Praz appelle «le romantisme noir ", de l'image du « vice anglais ", incarnée par le personnage du sadique policé et développée à partir de Swinburne ${ }^{10}$. Mais pourtant, à y regarder de plus près, voici quelqu'un qui, quoi qu'il en dise, se laisse convaincre facilement de suivre son compère au théâtre sanguinaire ( (Je viens vous chercher - Pourquoi ? - Vous savez bien...»), et dont la minutie des descriptions n'est peut-être pas innocente. À la fin de l'histoire, il offre son bras au pasteur et tous deux «redescendent, en silence, vers Grenade endormie ». Timide connivence ? La fascination du sang est peut-être contagieuse.

10 Comparée à Die Tamatensauce, la nouvelle Die Mamaloi va, semble-t-il, plus loin encore dans la subversion culturelle, dans la mesure où l'Européen, ici, n'est plus seulement fasciné, mais directement compromis dans la scène sauvage. Le narrateur est un Allemand venu s'installer voici quarante ans à Haïti pour y faire fortune et qui peut "s'enorgueillir, aujourd'hui, d'un des commerces les plus florissants de l'île ». Il y a quelque temps qu'il soupçonne sa jeune servante indigène, Adelaïde, de participer à des cérémonies secrètes du culte vaudou. Un soir, il entreprend de la suivre dans son escapade, autant, à l'en croire, par souci de faire respecter son autorité que par simple curiosité ( je ferai valoir ma volonté... J'appartiens à ce pays et j'ai le droit d'apprendre à le connaître dans toutes ses particularités »). Mais arrivé sur les lieux de la cérémonie rituelle, il se laisse bientôt lui aussi gagner par l'hystérie collective : «je sens qu'il faut que j'entre dans cette danse infernale de bêtes enragées... je hurle, je mords, je fais comme les autres..., sans la moindre retenue, j'écume et je vocifère, plus sauvagement et plus frénétiquement que personne» (c'est nous qui soulignons).

11 À la différence donc du pasteur anglais de Die Tamatensauce, l'Européen de Die Mamalai ne conserve pas une distance aristocratique-esthétique en face de la scène sauvage, mais s'y mêle comme acteur. Avec le recul, lui-même, sans porter de jugement moral ou exprimer un remords, interprète l'événement comme un moment d'égarement, d'abandon aux pulsions de l'inconscient : «Six mois se sont écoulés... j'ai l'impression que c'est un autre que moi qui a vécu ces événements dramatiques, qui sont si loin et étrangers à moi-même ». Quoi qu'il en soit, l'infraction va recevoir ici son châtiment. Après que, lors du soir fatal, dans l'hystérie "des sens déchaînés", les corps du narrateur et d'Adelaïde se sont étreints au cœur de la mêlée, celle-ci accouche bientôt d'un fils : quelques semaines plus tard, elle disparaît avec l'enfant ; craignant le pire, le narrateur part à sa recherche, mais arrive trop tard sur les lieux du sacrifice: son enfant est étranglé sous ses yeux par la prêtresse Marnaloi (Adelaïde elle-même) au milieu des cris « des démons noirs ». Ainsi la seconde scène sauvage est-elle en quelque sorte le prix de la première.

12 Une autre nouvelle, Der Spielkasten ${ }^{11}$, dont l'action est située au Tonkin, présente cette particularité, dans la mise en scène de la subversion culturelle, de renverser les rôles, entre l'indigène et l'Européen. C'est d'ailleurs moins dans l'intrigue elle-même, finalement banale (le prince Hong-Dok avait offert son amitié et ouvert sa maison à un officier de la Légion; mais celui-ci trahit sa confiance en séduisant l'une de ses femmes; Hong-Dok fait clouer le traitre et l'infidèle sur une croix et les abandonne, 
agonisants, sur un radeau qui va dériver pendant des jours le long du Fleuve Jaune...) que dans les mobiles qui sont prêtés aux acteurs que se joue le jeu des références, des décalages, des transgressions entre les codes culturels en présence. Il faut comprendre que Hong-Dok n'agit pas par jalousie : le jeune officier de la Légion lui eût révélé ses sentiments qu'il lui aurait sans hésiter offert aussitôt cette femme en gage d'amitié. Mais il n'a pu supporter l'idée d'avoir donné son estime à quelqu'un de vil et méprisable... La fourberie du séducteur l'a en fait conforté dans son dédain de la culture européenne, qu'il a appris à connaître au contact des colons français. Et la mort qu'il a choisie pour les coupables est pour lui « une manière de pousser jusqu'à l'absurde la conception européenne de la morale et de l'honneur $»^{12}$ :

«Ich habe den Seekadetten und Ot-Chen gekreuzigt... Warum? Ich habe sie - in flagranti erwischt». Das Wort gefiel ihm so gut, dass er es wiederholte. Er hatte es irgendwo gehört oder gelesen und es erschien ihm ungeheuer lächerlich, dass wir Europäer besonderen Wert darauf legen, wenn man einen Spitzbuden gerade bei seiner Tat entdeckt... Er sagte das mit einer wichtigen Hervorhebung, mit einer leicht herausklingenden Uebertreibung, die seine spöttische Verachtung so recht zeigte: «In f1agranti - Nicht wahr, da hat in Europa der betrogene Eheman das Recht, den Räuber seiner Ehre zu strafen ?... Ich habe ihn also bestraft. Und da er ein Christ ist, so hielt ich es für das beste, eine chrisliche Todesart zu wählen; ich glaubte, das würde ihm am meisten zusagen. War das recht? $»^{13}$

13 La scène sauvage ne ressort plus, comme dans Die Tarnatensauce et Die Marnaloi, d'une contamination de l'Européen par le monde exotique, mais, exactement à l'inverse, de celle de l'indigène par le monde occidental. La menace entraînée par ce désordre des valeurs n'est pas moins redoutable que dans le premier cas. C'est ce que laisse deviner la réaction du témoin-narrateur de l'histoire, un certain Edgard Widerhold, un Allemand établi dans le pays déjà avant l'arrivée des Français, et qui se sent indirectement responsable $\mathrm{du}$ drame, puisque c'est par son entremise que le légionnaire a connu Hong-Dok et a commencé de fréquenter sa maison...

14 L'Espagne, Haïti, le Tonkin - quelle que soit la géographie, l'exotisme, dans les premiers récits de H.H. Ewers, apparaît toujours comme une expérience des limites : il s'agit d'aller jusqu'au moment, jusqu'à l'endroit où commencent de vaciller convenances, convictions et certitudes culturelles ancrées. Ce qui ne signifie pas que le héros ewersien soit prêt, pour autant, à renier ses origines, à abdiquer son appartenance ethnique ou nationale. Dans la distance qu'il a mise et conquise entre lui et son pays, il entretient en vérité, avec son "chez soi ", une relation ambiguë, contradictoire, où entrent à la fois nostalgie et rejet.

15 Tandis que le narrateur de Die Tomatensauce le dit avec un humour au second degré («Right or wrong my contry!»), celui de Der Spielkasten, Edgar Widerhold, l'exprime avec la verve gouailleuse d'un vieil original :

Der Alte ist keiner, der Huna schreit. Er schimpft auf das Reich wie ein Rohrspatz. Uralt ist er, aber wenn er in Berlin lebte, müsste er zehnmal so alt werden, um all die Zeit abbrummen zu können, die ihm allein seine Majestätsbeleidigungen eintrügen. Auf Bismarck schimpfte er, weil er Sachsen leben liess und Böhmen nicht einsteckte, auf den dritten Kaiser, weil er sich Helgoland aufschwätzen liess für das ostafrikanische Reich... Das ist so mit ihm. Wenn ein Deutscher Gift und Galle spuckt über Kaiser und Reich, freut er sich und schimpft mit. Wenn ein Franzose Witze macht über uns, so lacht er, doch er revanchiert sich gleich und erzählt die neuesten Dummheiten des Gouverneurs in Saigon. Wenn aber ein Engländer es wagt, über unseren albernsten Konsul die harmloseste Bemerkung zu machen. wird er wütend... ${ }^{14}$ 
Quels que soient leurs sentiments, ni l'un ni l'autre n'envisagent de regagner benoîtement leurs pénates. Il n'y a finalement que le héros-narrateur de Die Marnaloi pour avoir succombé, un moment, à la tentation du retour. Au début de sa confession, il avoue qu'il fut autrefois, bien avant l'aventure vaudou, «dévoré par la nostalgie du pays natal » et que, quarante ans après avoir quitté l'Allemagne, il décida un jour de liquider tous ses biens pour aller vivre le reste de son âge au milieu des siens. Mais il ne put, nous dit-il, rester plus de trois semaines au pays, en raison du scandale suscité par les bruits qui couraient sur sa conduite aux Iles, en particulier son commerce coupable avec les fillettes indigènes. Il fut incapable de faire entendre à son frère qu'il n'avait fait, en la matière, que se conformer aux mœurs locales. Et il repartit donc, pour ménager l'honorable réputation de sa famille. Ce qui ne l'empêcha pas d'envoyer depuis ce temps à son frère, chaque mois, une somme appréciable pour contribuer à l'éducation de ses neveux. Et ce qui n'empêcha pas non plus ce frère de taire ses scrupules et d'accepter «sans trop de remords de conscience » les dites sommes des mains « d'un pécheur impénitent ». C'est donc finalement, à l'en croire, la société-mère elle-même qui a rejeté l'exilé repenti, qui a refusé d'ouvrir les bras au fils prodigue. Mais en fait, celui-ci sait très bien qu'il n'était pas prêt, au fond, à faire amende honorable, que son retour était une sorte de provocation. Pourtant, même après ce retour manqué, même après son irrémédiable compromission dans la scène sauvage, il ne rompt pas complètement avec ses origines. Preuve en est la prière qu'il fait à son correspondant - celui auquel il adresse sa confession et qui sera son " éditeur »: " Je tiendrai ma promesse et je relaterai tout scrupuleusement, ainsi que vous en exprimez le désir, en commençant depuis le début. Faites-en ce que vous voudrez, mais taisez mon nom, par égard pour ma parenté établie en Allemagne. Je voudrais leur épargner un nouveau scandale $»^{15}$.

17 Le voyageur ewersien installe une sorte de statu quo, entre lui et sa "patrie ». Il ne souhaite ni couper les ponts, ni réintégrer la maison - mais seulement faire reconnaître sa différence. L'aventure exotique est un destin singulier, aux deux sens du terme. Elle n'est pas le prétexte d'une pose anarchiste, mais l'occasion, pour l'individu qui s'y prête et y succombe, d'un dépassement de soi-même, d'une plongée vers ce que H.H. Ewers, dans son essai-manifeste sur E.A. Poe (1905), appelle « les profondeurs secrètes de l'âme ", dont l'exploitation est la vocation de l'art. « Un pionnier dans les terres inconnues de l'inconscient $»^{16}$ : telle est la définition de l'artiste selon H.H. Ewers, vérifiée, à ses yeux, chez ces maîtres que sont pour lui Poe, Hoffmann, Baudelaire et Villiers. L'aventure fantastique-exotique, dans les nouvelles que nous avons lues, répond en tout cas à l'intention de se situer dans cette lignée esthétique ${ }^{17}$.

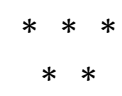

\section{La scène morbide}

18 Tout bascule avec l'événement de la Grande Guerre, qui, sous l'apparente continuité thématique, va changer radicalement le sens de l'exotisme ewersien, où l'aventure étrangement inquiétante glisse vers une simple histoire scabreuse aux relents 
xénophobes, qui marque la dégradation du talent de l'écrivain vers la «Trivialliteratur». La scène sauvage devient une scène morbide.

Il suffit de relire les premières lignes du roman Vampir. Ein verwildeter Roman in Fetzen und Farben, composé à cette époque et dont l'action est censée commencer précisément en 1914, pour mesurer la nouvelle évaluation de l'Ailleurs soutenue désormais par H.H. Ewers :

In dem Jabre, in dem die ganze Welt wahnsinnig wurde, war er hinausgezogen zum andern Mal. Er sagte immer: zum andern Mal; er zählte nieht, ob es das siebente Mal war, oder das zehnte, das zwölfte. Drei Jahre war er nun schon zu Hause geblieben, über drei Jahre schon in seiner alten Heimat: Europa. Er wusste wohl, dass er krank war; Europa machte ihn krank, die Heimat, die er liebte. Nach einem Jahre wusste er es selbst; nach zwei Jahren sahen es seine Freunde; nach drei Jahren merkten es alle, die mit ihm sprachen. Die Nerven, irgendwie... Aber er wusste auch, was ihn heilen mochte. Oder eigentlich nicht heilen. Wohl aber: ihm neue Kraft geben für neue Jahre in der Heimat. Wenn er die Gluten der Tropen trank, wenn er die Einsamkeiten der Wüsten atmete, wenn seine Schnsucht sich badete in den Unendlichkeiten aller Meere... ${ }^{18}$

L'enjeu de l'aventure exotique n'est plus une interrogation sur sa propre identité, mais une simple tentative de fuite, l'Ailleurs étant interprété, délibérément, comme une alternative salvatrice face au monde européen. Les Tropiques comme régénérescence. Cette vision implique, d'abord, une dégradation du héros. Bien qu'il porte le même nom, le Frank Braun de Vampir n'est plus le même que celui de Der Zauberlehrling (1909). Il a perdu sa superbe nietzschéenne qui faisait de lui le maître de la frénésie déchaînée dans la vallée de Val di Scodra, pour être ravalé, ici, au rang d'un "malade ». D'un névropathe exactement, puisque la suite du roman révélera que sa fébrilité provient en fait «d'une soif de sang " (sic) et de cruauté inavouée. Cette maladie n'est même pas singulière, dans la mesure où elle est présentée explicitement comme le symptôme de la frénésie collective qui s'est emparée des nations en guerre. Sans développer ici toutes les implications entraînées par cette «mythification » de l'Histoire, constatons simplement que tous les spectacles terrifiants que Frank Braun se plaît (et se complaît) maintenant à collectionner au cours de son voyage n'ont plus rien à voir avec ceux qui sont décrits dans les premières nouvelles de H.H. Ewers : il ne s'agit plus, ici, que de satisfaire un goût morbide.

Cette curiosité morbide assouvie dans l'exotisme va d'ailleurs de pair - ce n'est pas un hasard - avec une farouche conversion nationaliste. Autant le personnage du témoin, jusqu'ici, se gardait de tous les «hourras » (sic), autant notre Frank Braun s'enferre dans un chauvinisme exalté. Après avoir visité Cuba et le Mexique, il débarque aux États-Unis, où il devient rapidement l'animateur, le propagandiste zélé des cercles germanophiles, des comités de soutien à la cause allemande, dont les discours manient sans retenue tous les préjugés xénophobes éculés... Écho de la propre biographie de H.H. Ewers qui resta aux États-Unis durant toute la durée de la guerre (certains n'ont pas manqué de lui reprocher cette absence) et qui, à la demande, dit-on, de l'ambassadeur allemand, le comte Bernstorff, mit sa plume au service de la cause patriotique, ce qui lui valut d'être arrêté et incarcéré en 1917, au moment de l'entrée en guerre des Américains. H.H. Ewers ne rentra «chez-lui » qu'en 1920. Mais son ardeur nationaliste ne faiblit pas, au point même de le conduire vers l'allégeance au nationalsocialisme. L'écrivain cosmopolite et esthète, qui ne voulait s'adresser qu'à l'élite des esprits distingués - «die Kulturnation», disait-il - allait bientôt commettre Horst Wessel et Reiter in deutscher Nacht (1932). 

lation des faits et gestes du fossoyeur dans l'exercice de sa perversité - l'exotisme étant là pour " excuser " le réalisme complaisant du propos. L'histoire est en effet censée se dérouler dans cette région reculée de l'Illinois que les yankee ont baptisée «l'Égypte» en raison de sa population interlope - «un conglomérat de races inférieures : Croates, Slovaques, Hongrois, Tchèques, Valaches, Slovènes, Russes, Grecs, Italiens et Ukrainiens ${ }^{19}$. Le témoin, Jan Olieslagers, un flamand d'origine qui, depuis Je début de la guerre, "a travaillé pour la cause allemande» (un engagement qui illustre donc le bien-fondé des thèses pangermanistes !) est venu se réfugier dans cette région pour échapper à la prison au moment où les États-Unis rejoignent le camp des Alliés. Et il prend soin de marquer sa différence, d'affirmer sa distance devant les mœurs du pays qu'il visite : «Il ne fréquentait personne ». La conduite du fossoyeur ne l'émeut ni le scandalise : elle l'intrigue. Il la décrit « comme l'explorateur qui découvre dans la forêt tropicale une flore étrange et inconnue $»^{20}$. Que le lecteur se rassure: ses propres valeurs ne sont donc pas en cause, sa propre culture est intacte. Il est seulement convié à un spectacle scabreux. Carter $^{21}$, malgré son ostensible crudité, n'est pas davantage « subversive ». Un industriel américain, du nom de Phil Carter, dont l'entreprise a fait faillite, s'est suicidé : suivant la loi de l'État de Rhode Island, aux termes de laquelle le suicide est un crime religieux et social, le corps du défunt est jeté nuitamment au fond d'un fossé, après qu'un représentant de l'autorité lui a planté un pieu dans le cœur... La fille de l'industriel, contre promesse de ses faveurs, exige d'un de ses soupirants (le narrateur) d'aller 
déterrer le cadavre de son père et de le transporter dans une autre province pour lui donner une sépulture « honorable ». Et celui-ci d'exécuter la macabre besogne...

Une fois encore, et contrairement au cliché (déjà en vigueur dans la littérature de l'époque) qui fait des États-Unis l'univers-symbole de la modernité, de la civilisation la plus élaborée, l'Amérique, selon H.H. Ewers, est terre d'exotisme, c'est-à-dire un pays qui étale, comme l'écrit le héros, «des mœurs insensées et incroyables ». Le paradoxe veut qu'en l'occurrence, cet exotisme soit en fait le résultat d'un anachronisme culturel, dans la mesure où il est dit que la population du Rhode Island avait conservé « la vieille mentalité puritaine » et était restée fidèle à "l'ancienne loi anglaise sur le suicide ». Comme dans Die Marnaloi et Der Spielkasten, l'horreur est liée, finalement, au fanatisme religieux. Mais à la différence des deux premières nouvelles, il n'y a ici aucune compromission, contagion ou tentation, de la part du héros. En accomplissant l'exhumation promise - occasion d'une description «clinique » d'un cadavre dans un état de putréfaction avancé ! -, il ne fait pas œuvre perverse. Bien au contraire, son infraction à la législation du Rhode Island vient en vérité restaurer un ordre supérieur, celui de la morale "commune " (communément admise, en tout cas, dans les autres États de l'Union) qui débarrasse la loi chrétienne de son intransigeance et l'accommode à "l'honorabilité ». L'aventurier, naguère "pionnier dans les terres inconnues de l'inconscient », est devenu un redresseur de torts au nom de l'honnête charité! Il est d'autant plus dérisoire qu'il n'est même pas récompensé pour son dévouement : Eilenn Carter, si elle était prête à lui offrir son corps, aura la cruauté de repousser son amour. Mais elle n'a pas pour autant la superbe de la Belle Dame Sans Merci - figure-clef du fantastique décadent - qui sacrifie ses amants en incarnant à la fois le désir idéal et le désir de mort. C'est simplement, comme se l'avoue le narrateur, une jeune femme ambitieuse et soucieuse de respectabilité et qui était prête, pour cela, à monnayer «le seul bien qui lui restait ». Eilenn Carter se réduit à l'histoire banale d'un marché de dupes - d'un goût douteux.

On ne peut parler juste, à propos de H.H. Ewers, en prétendant occulter, ou minimiser, son allégeance au national-socialisme. Il ne s'agit pas de prêter à l'œuvre un poids qu'elle n'a pas, en accusant les quelques romans et recueils de nouvelles de l'écrivain d'avoir favorisé l'avènement de l'hitlérisme extrapolation grossière dénoncée à juste titre par Hubert Juin : «De grâce, que l'on s'abstienne d'écrire, comme certains, que ce même Ewers a vu l'épouvante nazie sortir de ses livres, et qu'il en fut ainsi parmi les créateurs. Des jeunes gens se sont suicidés, et il se trouvait des esprits fourbes pour soutenir que c'était la faute de Cocteau, ou de Gide, ou de Sartre ${ }^{22}$. Dont acte. Mais on ne saurait non plus soutenir, sous peine cette fois-ci, en prétendant défendre l'écrivain, d'accepter qu'il fût irresponsableet même si l'adhésion de H.H. Ewers fut éphémère, puisqu'il fut bientôt lui aussi, dès 1935, frappé de l'interdiction de publication - que cette compromission fut le fruit du hasard. Il faut au contraire, sans naïveté et arrièrepensée, la regarder dans la logique de l'œuvre.

Dire de H.H. Ewers, comme certains, qu'il ne relève que de la «Trivialliteratur» - c'està-dire d'une stratégie d'écriture délibérément conformiste - c'est ne pas avoir lu l'auteur de Das Grauen ou Die Besessenen, celui auquel l'éditeur munichois Georg Müller confie, à l'époque, la direction de la collection « Galerie der Phantasten ». Célébrer en lui, comme J.E. Poritzky, «le plus digne héritier de Villiers » ${ }^{23}$, sans discerner dans son. œuvre, c'est le surestimer. L'analyse des fictions exotiques de Ewers offre un angle de vue privilégié pour repérer le dérapage qui s'opère dans la production de l'écrivain, 
lorsque la Grande Guerre vient sonner le glas de l'esthétisme «fin-de-sièc1e ». Au bout du voyage, le globe-trotter se compromet. L'insolence devient arrogance.

\section{NOTES}

1. La première édition allemande de l'œuvre d'E.A. Poe date de 1901 (traduction de H. et A. Moeller-Bruch, 10 volumes, Minden).

2. Wolfgang Reif, Zivilisationsflucht und literarische Wunschräume. Der exotistische Roman im ersten Viertel des 20. Jahrhunderts, Metzler Verlag, Stuttgart, 1975, pp. 16-21.

3. Soit l'avertissement dû à A. Landsberger: «Lebendige und Tote reichen sich zu einem farbenfrohen und doch sa düsteren Tanze hier die Hände und legen Zeugnis dafür ab, dass trotz allen gegenteiligen Geredes in allen Breitengraden und Zonen, unter den Tropenpalmen Zentralafrikas und unter den Föhren des höchsten Nordens, in Indiens Dschungeln wie auf der britischen Nebelinsel Mensch immer Mensch geblieben ist und bleiben wird» (p. 12). C'est en vertu de ce principe que voisinent, au sommaire du recueil, R. Kipling, F. Gerstäcker et Villiers de l'Isle-Adam !

4. Précisons que nous entendons par « exotisme » un décor décrit et valorisé, à l'intérieur du texte lui-même, comme un Ailleurs. Il va de soi qu'aucune géographie n'est, a priori, « exotique ».

5. München, 1922.

6. München, 1920.

7. In : Das Grauen, G. Müller, München, 1908.

8. Idem., p. 21.

9. Hanns Heinz Ewers. Die Geschichte seiner Entwicklung, Leipzig, 1922, p. 27 sq.

10. Mario Praz, La chair, la mort et le diable dans la littérature du xxe siècle. Le romantisme noir, Denoël, Paris, p. 343 sq.

11. In : Die Besessenen, op. cit.

12. Cité d'après la réédition Geschichten des Grauens, Herbig, München, p. 142.

13. Idem., p. 141.

14. Ibid., pp. 108-109.

15. In : Das Grauen, op. cit., p. 241.

16. Hanns Heinz Ewers, Edgar Allan Poe, Schuster \& Loefner, Berlin, 1905.

17. «In grauer Nebelwolke träumt vor uns das gewaltige Land des Unbewussten... Und viele, viele gehen schmählich zugrunde, ohne je einen Blick hinter die Wolken zu werfen. Ganz wenigen aber gelingt die Fahrt. Sie finden ein Neuland, entdecken es für die Kultur: sie haben die Grenzen des Bewusstseins ein Stück weiter hinausgeschoben. Die Künstler sind diese ersten Entdecker» (Idem., pp. 19-21).

18. Op. cit., p. 11.

«Was war sein Vaterland? Seine Heimat, das war gewiss, war Europa. In Wien war er zu Hause, in Berlin, in München und am Rhein. Aber nicht weniger in der Bretagne, in der Provence, in Paris. Und in Italien - überall! In Andalusien auch und in der Stadt, die den Prado barg. Und in Stockholm, in Pest, in Zürich und Antwerpen... International? Nein? So hatte er nie empfunden. Aber es gab über allen Völkern ein anderes Volk, höher, edler und grösser. Die Kulturnation hatte er es genannt - ihr gehörte alles an, was hinausflog über die Massen. Und er kannte sie gut, 
fand ihre Bürger überall in der Welt. Es war da, dieses Volk, ganz gewiss, ohne jeden Zweifel» (Idem., p. 23).

19. Idem., p. 7.

20. Ibid., p. 40. «Der Staatsanwalt hätte von Verbrechen gesprochen, der Mediziner von Wahnsinn. Für Jan Olieslagers war es weder das eine nocb das andere. Der Gedanke, die Taten Stephes moralisch oder gar ästhetisch zu werten, kam ihm gar nicht...» (Ibid., p. 40).

21. In : Von sieben Meeren. op. cit.

22. Les Lettres françaises du 29 juillet 1970.

23. «Ich weiss bei uns keinen würdigeren Erben dieses Dichters - Villiers - als Hanns Heinz Ewers... der in seinen mit Grausamkeit und Pracht gemischten Geschichten auf unseren Nerven spielt wie Kreisler auf seiner Geige» (J.E. Poritzky, Dämonische Dichter. München, 1921, p. 104).

\section{RÉSUMÉS}

En recherchant, dans les fictions dites « exotiques » de H.H. Ewers, comment s'opère l'évaluation de l'Ailleurs, comment fonctionne le jeu des références, des décalages, des transgressions entre les codes culturels, se dégagent, autour de la césure de la Grande Guerre, du « retour chez soi » de l'écrivain globe-trotter et de sa conversion nationaliste, deux modes d'écriture qui résument bien toute la dérive de H.H. Ewers, de l'esthétisme décadent vers la «Trivialliteratur».

L'exotisme offre d'abord une scène sauvage: le moment, le lieu où se déchaîne, devant l'Européen, une frénésie reconnue sans doute d'abord comme "indigène », mais dans laquelle il se trouve bientôt (involontairement?) impliqué - contagion où vacillent les certitudes axiologiques les mieux partagées.

À la scène sauvage succède, dans les derniers récits de H.H. Ewers, la scène morbide : pantomime macabre offerte à la curiosité détachée et arrogante d'un témoin-narrateur ravalé au rang de voyeur et conforté dans son assurance de supériorité culturelle.

Le retour chez soi, après l'aventure exotique, n'est pas sans risque. La compromission avec le national-socialisme, pour H.H. Ewers, en fut en quelque sorte le prix.

Sucht man in den sogenannten "exotischen» Fiktionen von H.H. Ewers wie das Anderssein bewertet wird, wie das Spiel der Verweise, Brüche und das Ineinandergehen der Kulturen inszeniert wird, so lässt sich, var und nach dem 1. Weltkrieg, der Heimkehr des Schriftstellers und seiner Bekehrung zum Chauvinismus, eine Zäsur feststellen, in deren Folge sich zwei Schreibarten herausbildeten, die den Verfall seines Talentes, von dem dekadenten Asthetizismus bis zur Trivialliteratur, konkretisieren.

Der Exotismus wird uns zunächst als eine wilde Bühne vorgeführt: als ein Moment und ein Ort, wo unter den Augen des Europäers ein frenetisches Schauspiel entfesselt wird, das zunächst wohl als etwas Fremdes wahrgenommen wird, in das er aber bard (ob er nun will oder nicht) hineingezogen wird - nach einer Art Vergiftung, in der alle ethischen, allgemeingültigen Begriffe ins Schwanken geraten.

Auf die wilde Bühne folgt dann, in den letzten Erzählungen von H.H. Ewers, die morbide Bühne: ein makabres Spiel, das der distanzierten und arroganten Neugier eines voyeurhaften Zeugen geboten wird, der dadurch von seiner kulturellen Ueberlegenheit noch überzeugter ist.

Die Rückkehr in die Heimat, nach dem exotischen Abenteuer, ist nicht ganz gefahrlos. Die 
Tatsache, dass H.H. Ewers zeitweise dem Nationalsozialismus huldigte, war der Preis seiner Heimkehr.

\section{AUTEUR}

JEAN-JACQUES POLLET

Université de Lille III 